\title{
The Variational Iterations Method for the Three- dimensional Equations Analysis of Mathematical Physics and the Solution Visualization with its Help*
}

\author{
Aleksey Tebyakin, Irina Papkova ${ }^{[0000-0003-4062-1437]}$, and \\ Vadim Krysko ${ }^{[0000-0002-4914-764 X]}$ \\ Yuri Gagarin State Technical University of Saratov, \\ 77 Politechnicheskaya street, Saratov, Russia, 410054 \\ prototype9235@mail.ru, ikravzova@mail.ru, tak@san.ru
}

\begin{abstract}
The aim of the work is to use the variational iterations method to study the three-dimensional equations of mathematical physics and visualize the solutions obtained on its basis and the 3DsMAX software package. An analytical solution of the three-dimensional Poisson equations is obtained for the first time. The method is based on the Fourier idea of variables separation with the subsequent application of the Bubnov-Galerkin method for reducing partial differential equations to ordinary differential equations, which in the Western scientific literature has become known as the generalized Kantorovich method, and in the Eastern European literature has known as the variational iterations method. This solution is compared with the numerical solution of the three-dimensional Poisson equation by the finite differences method of the second accuracy order and the finite element method for two finite element types: tetrahedra and cubic elements, which is a generalized Kantorovich method, based on the solution of the three-dimensional stationary differential heat equation. As the method study, a set of numerical methods was used. For the results reliability, the convergence of the solutions by the partition step is checked. The results comparison with a change in the geometric parameters of the heat equation is made and a conclusion is drawn on the solutions reliability obtained. Solutions visualization using the 3Ds $\max$ program is also implemented.
\end{abstract}

Keywords: Heat Equation, Method of Variational Iterations, Analytical Solution, Finite Difference Method.

Copyright (C) 2020 for this paper by its authors. Use permitted under Creative Commons License Attribution 4.0 International (CC BY 4.0).

* This work was supported by the Russian Science Foundation project RNF № 19-19-00215. 


\section{Introduction. The history of the issue}

In the work, to solve the three-dimensional equations of mathematical physics (Poisson equation), a method is used that is a generalization of the Fourier variables separation method and is known in the literature as the variational iterations method. This method is based on the idea of reducing partial differential equations to ordinary differential equations for elliptic equations - this is the Kantorovich method with the subsequent refinement of the function with respect to the desired variables - the variational iterations method. In Western European literature, this method is called the extended Kantorovich method, in Eastern European literature it is called the method of variational iterations (MVI). It allows you to get an analytically accurate solution at every step of the iterative procedure.

This method was first proposed and used in 1933 by T.E. Shunk [1] for calculating the bending of cylindrical panels. Unfortunately, the work was not noticed, and the method was rediscovered again in 1964 E.E. Zhukov [2], who used it to calculate thin rectangular plates. In the future, MVI was widely used by many researchers in solving problems of the shells and plates theory (a bibliography on this subject is presented in [3]). The method justification for the class of equations described by positive definite operators is given in [4].

The variational iterations method (extended Kantorovich method) over the past half century has been used to solve problems of statics, stability, natural frequencies determination and dynamics. A fairly complete review of Western publications in this area can be found in $[5,6]$. In the USSR and Russia, this method was mainly used in the works of V.A. Krysko and his students. For the first time, this scientific group used the approach in 1968 to study the bending of flexible orthotropic plates [7], and approach got its name the variational iterations method in the work [8] 1970, devoted to the numerical study of flexible plates and comparison with experimental data. Subsequently, scientists of this group used the variational iterations method to solve geometrically and physically nonlinear problems in the theory of shells and plates [9, 10], in problems of designing optimal plates [11-12], and on other topics [13-16].

In this paper, this method is first used for the three-dimensional Poisson equation, which shows the relevance of this work. To implement these methods and obtain the final solution, the MATLAB application package is used. As a visualization of the obtained data, a software utility made for the Autodesk 3Ds Max platform is used. 3Ds Max has extensive tools for creating a diverse in form and complexity of threedimensional computer models, real or fantastic objects of the surrounding world, using a variety of techniques and mechanisms.

\section{$2 \quad$ Variational iterations method}

We apply MVI to find an analytical solution for the differential equation. Consider the stationary differential heat equation for a three-dimensional body (1). 


$$
\begin{gathered}
\kappa \nabla T(x, y, z)+f(x, y, z)=0 \\
\nabla T=\frac{\partial^{2} T}{\partial x^{2}}+\frac{\partial^{2} T}{\partial y^{2}}+\frac{\partial^{2} T}{\partial z^{2}},
\end{gathered}
$$

where $T(x, y, z)$ - temperature field, $f(x, y, z)$ - the internal heat source density, which is considered to be given, $\kappa$ - thermal diffusivity,

$$
\Omega=\left\{T_{\min } \leq T(x, y, z) \leq T_{\max }\right\},
$$

$(x, y, z) \in \Omega=(0, a) \times(0, b) \times(0, c)$ - temperature distribution area; $\partial \Omega$ - border area.

Edge conditions of the 1 st kind

$$
\left.T\right|_{\partial \Omega}=t_{\Gamma}=\text { const }
$$

Edge conditions of the 2 nd kind

$$
-\lambda\left[\frac{\partial T}{\partial n}\right]_{n=-0}=q_{r}
$$

where $q_{r}$ - is the given value of the heat flux on the surface. The notation $\mathrm{n}=-0$ emphasizes that the values are calculated inside the object infinitely close to its surface.

Edge conditions of the 3rd kind

$$
-\lambda\left[\frac{\partial T}{\partial n}\right]_{n=-0}=\alpha\left(t_{w}-t_{f}\right)
$$

The proportionality coefficient $\alpha$ in (4) is called the heat transfer coefficient and is a measure of the convective heat transfer intensity between the surface and the heat carrier (for the sake of brevity, this is called heat transfer), $t_{w}-t_{f}$ is the temperature thrust, the temperature difference between the wall (surface) and the environment.

We apply the method of variational iterations in a first approximation to the heat equation taking into account the boundary conditions (2).

In the variational iterations method, it is assumed that the function consists of the one-dimensional functions product with respect to each variable:

$$
T(x, y, z)=A(x) B(y) C(z)
$$

We introduce a three-dimensional bounded Hilbert space $H\{(0,1) \times(0,1) \times(0,1)\}$ where each space function has a continuous derivative of at least second order. We introduce a three-dimensional bounded Hilbert space - where each function of this space has a continuous derivative of at least second order. To find a solution, we use condition (3) so that:

$$
\iiint_{H}(\kappa \nabla T(x, y, z)+f(x, y, z)) \cdot T(x, y, z) d x d y d z=0
$$

Substituting (10) into (11) we get

$$
\begin{array}{r}
\int_{0}^{a} \int_{0}^{b} \int_{0}^{c}\left[\kappa \left(\frac{\partial^{2} A(x)}{\partial x^{2}} B(y) C(z)+\frac{\partial^{2} B(x)}{\partial x^{2}} A(y) C(z)+\right.\right. \\
\left.\left.+\frac{\partial^{2} C(z)}{\partial x^{2}} A(x) B(y)\right)+f(x, y, z)\right] A(x) B(y) C(z) d x d y d z=0
\end{array}
$$


Subsequently, from equation (7), the functions $A(x), B(y), C(z)$ are found. Since the method implements an iterative process, it is required to set the initial approximation. For example, if you want to find the function $C(z)$, then you need to specify any functions $A(x)$ and $B(y)$ satisfying the conditions of the Hilbert space $H$.

The result is an inhomogeneous ordinary differential equation with constant coefficients with respect to the function $C(z)$.

$$
C^{\prime \prime}(z)+\frac{R_{2}}{R_{1}} C(z)=\frac{R_{3}}{R_{1}},
$$

where

$$
\begin{gathered}
R_{1}=\kappa \int_{0}^{a} \int_{0}^{b} A^{2}(x) B^{2}(y) d x d y \\
R_{2}=\kappa \int_{0}^{a} \int_{0}^{b}\left(\frac{\partial^{2} A(x)}{\partial x^{2}} B(y)+\frac{\partial^{2} B(y)}{\partial y^{2}} A(x)\right) A(x) B(y) d x d y \\
R_{3}=\int_{0}^{a} \int_{0}^{b} f(x, y, z) A(x) B(y) d x d y .
\end{gathered}
$$

When solving an inhomogeneous differential equation, the boundary conditions (2) are taken into account. The result of the solution is the function $C(z)$. The functions $A(x)$ and $B(y)$ are found in a similar way, but already using the found functions. After all the functions for each variable are found, we obtain an approximate analytical solution. Only the first iteration was carried out. Next you can carry out this procedure as many times as you like and get an increasingly accurate solution to equation (1). Further, this problem will be considered using the numerical method.

\section{$3 \quad$ Finite difference method}

To compare and analyze the results using the methods described above, the finite difference method with second-order approximation is used. We write the difference heat equation:

$$
\begin{aligned}
& T_{i, j, k}^{(s+1)}=\left(\kappa \frac{T_{i+1, j, k}^{(s)}+T_{i-1, j, k}^{(s)}}{h_{x}^{2}}+\kappa \frac{T_{i, j+1, k}^{(s)}+T_{i, j-1, k}^{(s)}}{h_{y}^{2}}+\right. \\
& \left.+\kappa \frac{T_{i, j, k+1}^{(s)}+T_{i, j, k-1}^{(s)}}{h_{z}^{2}}+f(x, y, z)\right) /\left(\frac{2}{h_{x}^{2}}+\frac{2}{h_{y}^{2}}+\frac{2}{h_{z}^{2}}\right) \\
& \max \left|T_{i, j, k}^{(s+1)}-T_{i, j, k}^{(s)}\right|>\varepsilon, \text { где } \varepsilon>0
\end{aligned}
$$

We attach the boundary conditions of the 1st kind (2). When the iteration process starts, the array of the function $T^{(0)}{ }_{i, j, k}$ is filled with zeros. After using formula (10) over the entire array, we obtain a new scalar field $T^{(1)}{ }_{i, j, k}$. We carry out this procedure while (11) is satisfied, where $e$ is the maximum difference between the values of 
adjacent iterations. A sufficiently small number is chosen as $\varepsilon$ in order to approach the exact solution. We apply MVI to find an analytical solution for the differential equation.

\section{$4 \quad$ Results analysis}

For the heat equation (1), taking into account the boundary conditions (2), we compare the solutions that were obtained by the numerical method (finite difference method) and the analytical method (variational iteration method in a first approximation). The results are presented in table 1 . Three cases were considered: $a=$ $b=c=1 ; a=3, b=c=1 ; a=5, b=c=1$. As the results, the maximum values of the functions obtained in the solution process were taken. The difference between the results obtained by analytical and numerical methods is $5.34 \%$.

Table 1. Numerical experiment results.

\begin{tabular}{|c|c|c|c|}
\hline MVI & $\mathrm{a}=\mathrm{b}=\mathrm{c}=1$ & $\mathrm{a}=3, \mathrm{~b}=\mathrm{c}=1$ & $a=5, b=c=1$ \\
\hline Maximum $\{\mathrm{A}(\mathrm{x})\}$ & 0.493542 & 0.595027 & 0.594314 \\
\hline Maximum $\{\mathrm{B}(\mathrm{y})\}$ & 0.409915 & 0.396978 & 0.395888 \\
\hline Maximum $\{\mathrm{C}(\mathrm{z})\}$ & 29.377056 & 3.536978 & 1.281865 \\
\hline $\begin{array}{c}\text { Maximum } \\
\{\mathrm{T}(\mathrm{x}, \mathrm{y}, \mathrm{z})=\mathrm{A}(\mathrm{x}) \mathrm{B}(\mathrm{y}) \mathrm{C} \\
(\mathrm{z})\}\end{array}$ & 5.9432295 & 0.835481 & 0.301600 \\
\hline $\begin{array}{l}\text { Finite difference } \\
\text { method }\end{array}$ & $21 \times 21 \times 21$ & $21 \times 21 \times 21$ & $21 \times 21 \times 21$ \\
\hline $\begin{array}{c}\text { Maximum } \\
\{\mathrm{T}(\mathrm{x}, \mathrm{y}, \mathrm{z})=\mathrm{A}(\mathrm{x}) \mathrm{B}(\mathrm{y}) \mathrm{C} \\
(\mathrm{z})\}\end{array}$ & 5.599181 & 0.814254 & 0.294009 \\
\hline
\end{tabular}

For MVI, table 1 presents a data set for each of the functions obtained by the method lying on its axis. The solution obtained by this method is shown in fig. 1, 2, 3 . Here is shown the surface of the temperature distribution in the middle of the region relative to the $\mathrm{Oz}$ axis. To build the surface, we used a special program written on the 3Ds Max platform. 


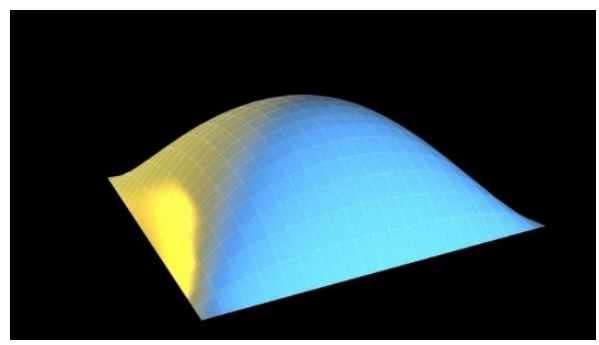

Fig. 1. Visualization of the numerical solution for the median plane

$$
\mathrm{a}=\mathrm{b}=\mathrm{c}=1
$$

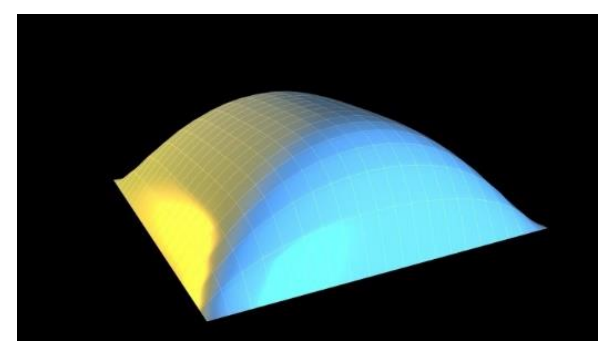

Fig. 2. Visualization of the numerical solution for the median plane

$$
\mathrm{a}=3 ; \mathrm{b}=\mathrm{c}=1
$$

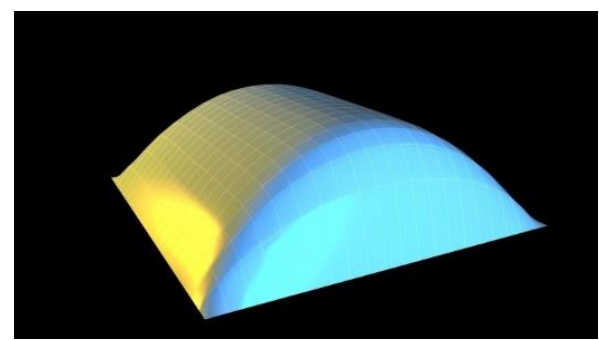

Fig. 3. Visualization of the numerical solution for the median plane

$$
\mathrm{a}=5 ; \mathrm{b}=\mathrm{c}=1
$$

\section{Conclusion}

1. For the first time, to obtain an analytical solution of a three-dimensional partial differential equation, the variational iterations method is used, which is based on the Kantorovich method - reduction of partial differential equations to ordinary differential equations. 
2. This method has high accuracy even in the first approximation, as evidenced by the solution of the same equations obtained by the finite differences method of the second accuracy order for a the first kind boundary value problem.

3. The results of a numerical solution using the variational iteration method were visualized using a software package written on the 3Ds Max platform.

\section{References}

1. Schunk, T.E.: Zur Knienfestigkeit schwach gekrummter zylindrischer Schalen. Arch., IV, $394-414,(1933)$

2. Zhukov E.E.: Variational technique of successive approximations as applied to the calculation of thin rectangular plates. - V kn.: Raschet tonkostennyh prostranstvennyh konstrukcij. Stroyizdat. Moscow. (1964).

3. Krysko, V.A.: Nonlinear statics and dynamics of inhomogeneous shells. Saratov University. Saratov. (1976)

4. Kirichenko, V.F., Krysko, V.A: The method of variational iterations in the theory of plates and shells and its substantiation. Applied Mechanics 17(4), 71-76 (1981)

5. Singhatanadgid, P., Singhanart, T.: The Kantorovich method applied to bending, buckling, vibration, and 3Ds stress analyses of plates: A literature review. Mechanics of Advanced Materials and Structures. 26(2), 170-188 (2019)

6. Banerjee, M.M., Mazumdar, J. A.: Review of Methods for Linear and Nonlinear Vibration Analysis of Plates and Shells. 2016. Procedia Engineering. 144, c. 493-503

7. Krysko, V.A., Amelchenko, V.V.: To the calculation of flexible orthotropic plates by the modified Vlasov-Kantorovich method using a digital computer. For technical progress 7 , 13-15 (1968)

8. Krysko, V.A., Amelchenko, V.V.: Calculation of flexible plates by the variational method of iterations and comparison with experimental data. In: 3rd interuniversity. Conf. p. 6266. SSTU, Saratov (1970)

9. Krysko, V.A., Fedorova, A.G.: Problems of dynamics for elastic-plastic flexible shallow shells. Applied Mechanics 15(2), 71-76 (1979)

10. Krysko, V.A., Fedorova, A.G.: Stability of shallow shells under the action of local loads, taking into account geometric and physical nonlinearities. Izv. Universities. Building and architecture 5, 24-28 (1978)

11. Krysko, V.A., Bochkarev, V.V.: Design of plates and shells close to optimal in weight taking into account the temperature effect. Applied Mechanics 17(11), $54-59$ (1981)

12. Krysko, V.A., Bochkarev, V.V.: Optimal design of plates and shells taking into account physical nonlinearity. Applied Mechanics 18(7), 52-57 (1982)

13. Krysko, V.A., Pavlov, S.P.: The problem of optimal control of the natural frequency of inhomogeneous shells. Applied Mechanics 18(4), 41-47 (1982)

14. Krysko, V.A., Bochkarev, V.V.: Stress-strain state of a plate of variable thickness with a flat front surface. Izv. universities. Mechanical engineering 12, 231-235 (1984)

15. Krysko, V.A., Kirichenko, V.F., Surova, N.S. On projection methods for solving problems in the theory of flexible shallow anisotropic multilayer shells. In: Proceedings of the II All-Union. conf. on scientific and technological products made of composite materials. pp. 99-107, Yerevan (1984)

16. Krysko, V.A., Zhigalov, M.V.: The method of linearization and order reduction of systems of differential equations in nonlinear mechanics of inhomogeneous structures. In.: IV Int. conf. on the mechanics of inhomogeneous structures, pp. 356. Ternopil (1995) 